\title{
Guest editorial to the special issue on inductive logic programming, mining and learning in graphs and statistical relational learning
}

\author{
Hendrik Blockeel · Karsten Borgwardt • Luc De Raedt • \\ Pedro Domingos $\cdot$ Kristian Kersting $\cdot$ Xifeng Yan
}

Received: 5 June 2010 / Revised: 5 June 2010 / Accepted: 12 June 2010 / Published online: 9 July 2010 (C) The Author(s) 2010. This article is published with open access at Springerlink.com

In 2009, three international conferences/workshops on learning from relational, graph-based and probabilistic data were co-located: ILP-2009, the 19th International Conference on Inductive Logic Programming; MLG-2009, the 7th International Workshop on Mining and Learning with Graphs; and SRL-2009, the International Workshop on Statistical Relational Learning. These events were organized in Leuven, Belgium, on July 2-4, 2009.

The ILP conference series has been the premier forum for work on logic-based approaches to learning for almost two decades and has recently reached out to other forms of relational learning and to probabilistic approaches. The MLG workshop series focuses on graph-based approaches to machine learning and data mining while the SRL workshop series focuses on statistical inference and learning with relational and first-order logical

H. Blockeel · L. De Raedt $(\bowtie)$

Department of Computer Science, Katholieke Universiteit Leuven, Leuven, Belgium

e-mail: luc.deraedt@cs.kuleuven.be

H. Blockeel

e-mail: hendrik.blockeel@cs.kuleuven.be

K. Borgwardt

Machine Learning and Computational Biology Research Group, Max Planck Institutes Tübingen,

Spemannstr. 38, 72076 Tübingen, Germany

e-mail: karsten.borgwardt@tuebingen.mpg.de

P. Domingos

Department of Computer Science and Engineering, University of Washington, Seattle, WA 98185, USA

K. Kersting

Knowledge Discovery Department, Fraunhofer IAIS, Schloss Birlinghoven, 53754 Sankt Augustin, Germany

e-mail: kristian.kersting@iais.fraunhofer.de

X. Yan

Computer Science Department, University of California, Santa Barbara, CA 93106, USA

e-mail: xyan@cs.ucsb.edu 
representations. The combination of probability theory with relational (or first-order logic) knowledge representation has been the subject of much recent research. While the three series clearly have their own identity, there is a significant overlap in the topics covered by each of them. In particular, the problem they study is essentially the same: learning from structured data involving multiple objects as well as the relationships that hold amongst them. The aim of the colocation was to increase the interaction between the three communities. The format of the joint event stimulated such interaction by providing joint invited speakers and tutorials, joint sessions and poster sessions, and ample time and space for discussions in smaller groups, in addition to the regular programs of the three events. For SRL and MLG, there were no formal proceedings though a post-conference proceedings has been published for ILP, in Volume 5989 of Springer's Lecture Notes in Artificial Intelligence series. In addition, the authors of eight papers, selected at the event, were invited to submit an extended version of their paper to the special issue, and of these, five papers were accepted after two rounds or reviewing.

The paper "Effective Feature Construction by Maximum Common Subgraph Sampling" by Leander Schietgat, Fabrizio Costa, Jan Ramon and Luc De Raedt proposes a novel method to construct features from graphs in a molecular setting. It is based on an algorithm that computes the maximum common subgraph using the block-and-bridge preserving notion of subgraph isomorphism, which runs in polynomial time on outerplanar graphs. The operation is then applied to randomly sampled pairs of molecules. The resulting approach is experimentally evaluated and shown to yield excellent performance.

In "Block-Wise Construction of Tree-like Relational Features with Monotone Reducibility and Redundancy", Ondřej Kuželka and Filip Železný contribute an efficient propositionalization technique following a novel block-wise approach to construct a feature set, by identifying smaller conjunctions ('building blocks') out of which all features can be composed. Unlike traditional level-wise approaches which preserve the monotonicity of frequency, the block-wise approach preserves monotonicity of feature reducibility and redundancy, which are important in the context of classification learning and which serves as the basis for pruning the search. The resulting approach scales to features including tens of first-order atoms and compares favorably with alternative techniques in inductive logic programming.

The paper "Efficient mining for structurally diverse subgraph patterns in large molecular databases" by Andreas Maunz, Christoph Helma and Stefan Kramer presents a graph mining approach for efficiently retrieving tree-shaped subgraph descriptors under minimum frequency and significance constraints from a database of graphs. Key to their approach is the definition of classes of fragments that share a common backbone. As their method is able to optimize structural inter-feature entropy, it detects sets of structurally diverse subgraph patterns. In experiments on large-scale graph data, their method allows to significantly reduce the feature set size and computational runtime of pattern mining.

In the article titled "Relational Information Gain", Marco Lippi, Manfred Jaeger, Paolo Frasconi and Andrea Passerini present a novel way of estimating the potential information gain that a new literal may yield when added to a rule. Already in the early years of relational learning, it has been recognized that when a literal is added to the condition part of a rule, this literal may be useful even if it does not change the coverage of the rule, because it may be introducing new variables that are relevant for classification. Up till now, this issue has been addressed by giving a bonus to literals introducing new variables, or by looking ahead in the search space. With the Relational Information Gain measure, Lippi et al. provide a principled way of estimating how informative the introduced variables may at best be for any refinement of the current rule, without actually enumerating these refinements. They show empirically that the estimate is both efficient and accurate. 
The paper "Constraint-based Probabilistic Modeling for Statistical Abduction" by Taisuke Sato, Masakazu Ishihata, and Katsumi Inoue introduces constraint-based probabilistic modeling (CBPM) that unifies the two most prominent paradigms for statistical relational modeling: feature-based approaches such as Markov logic networks and logic-based approaches such as ProbLog. The idea is to constrain a set of independent propositional variables (the ground atoms) by a knowledge base $\mathrm{KB}$ so that their joint distribution is the intended one. In doing so, CBPM can be used to express both generative models such as PCFGs and discriminative models such as CRFs as well as to perform logical inference based on KB. Additionally, Sato et al. prove the existence of CBPMs in infinite domains, derive a novel EM algorithm for learning the parameters of CBPMs, and demonstrate how to use CBPMs for statistical abduction

We are grateful to the authors, the reviewers, as well as to the Machine Learning journal for making this special issue possible.

Open Access This article is distributed under the terms of the Creative Commons Attribution Noncommercial License which permits any noncommercial use, distribution, and reproduction in any medium, provided the original author(s) and source are credited. 